UDC81-11:159.946.3]:1

\title{
SYNERGISM AND SUPERVENIENCE IN COGNITIVE PROCESSES
}

\author{
Alla Kalyta \\ National Technical University of Ukraine "Igor Sikorsky Kyiv Polytechnic Institute", \\ Kyiv, Ukraine \\ kalitanewadd@gmail.com
}

Oleksandr Klymenyuk

International University of Finance,

Kyiv, Ukraine

san-aleksandr@ukr.net

Received March, 19, 2020; Accepted May, 07, 2020

\begin{abstract}
The paper presents the results of the authors' original philosophical and linguistic consideration of the links between synergism and supervenience during cognitive processes of the human's speakingand-thinking activities. Within the framework of an interdisciplinary approach to the study of linguistic phenomena, the authors describe the influence of a multifactorial nature of cause-and-effect relationships on psycho-energetic features of the cognition mechanism functioning. On the basis of previously stated postulates, the authors have formed a systemic cybernetic model of the structure and complex interaction of causing factors that affect the self-development of cognitive processes in the human's psyche. The paper outlines the specificity of cognitive processes taking place in the four spheres (existential, mental, transcendental, consciousness) of the individual's spiritual being as well as reveals the essence of methodological potential of the cybernetic model proposed by the authors as a universal interdisciplinary tool for planning and carrying out lingua-cognitive research. Practical recommendations are given for the system planning of a new topic of interdisciplinary lingual-andenergetic research of the features of cognitive processes of speaking-and-thinking self-development, materialized in the person's speech and communicative behavior.
\end{abstract}

Key words: synergism, supervenience, cognition, self-development, causing factors, methodological tools, research in the sphere of energetics.

\section{Introduction}

The effectiveness of the use of synergetic analysis as a direction of present-day methodology of interdisciplinary research of self-developing cognitive processes and lingual-cognitive phenomena has been thoroughly presented in a number of works (Klymenyuk 2005: 160-163; Klymenyuk 2010: 97-126). These works take into account (Kalyta 2016: 122-137; Kalyta \& Klymenyuk) the existence of a close connection between synergy and supervenience in cognitive phenomena of individual's speakingand-thinking and thinking-and-acting processes. Without consideration of such a connection, any interdisciplinary description of energetic regularities and mechanisms of oral speech prosodic organization will be incomplete.

The need for such an understanding was exacerbated when it was necessary to define the leading factors influencing the course of a specifically studied cognitive 
process or phenomenon. But, until now, for a far from simple, due to the multifactorial (multifarious) nature of the synergetic cognitive phenomena themselves, the procedure for analytical substantiation of a complex of leading factors that basically determine the results of these phenomena self-development, the presence of a highly efficient conceptual paradigm that would describe the connection between synergism and supervenience is an acute and urgent problem.

At the same time, the long-term results of our theoretical and experimental lingual-and-cognitive research indicate not only the absence of the required paradigm, but also the frequent attempts made by the scientists in cognitive field to avoid mentioning or referring to the terms of synergism and supervenience in their obviously interdisciplinary works.

Therefore, the aim of the undertaken research is a theoretical paradigmatic substantiation of the mechanisms of the relationship between synergism and supervenience in cognition, which can serve as a methodological tool for conducting interdisciplinary lingual-and-cognitive research.

\section{Research methodology}

Given the interdisciplinary nature of the undertaken search, we will consider this problem from the point of high-level abstraction, reaching beyond purely linguistic problems, if necessary.

We shall note, first of all, that in terms of cognitive science, their direct relation to multifactorial phenomena having the nature of open nonlinear systems is common for both synergism and supervenience.

Let us start the analysis, as it should be, with the fact that the main feature of these concepts' differentiation in scientific descriptions of the surrounding reality should be their functional loading. Thus, if the term "synergism" clearly refers to the energetic aspect of cognition, since it indicates that the sources of its selfdevelopment are of a different nature, then the concept of "supervenience" is used to mark the complex causative-consecutive relationships that take place in any cognitive phenomena.

Hence, it is clear that the scope of our consideration should be framed within the sphere of existing scientific ideas about the reasons, regularities and mechanisms of various types of energy fluctuations in cognitive processes of an individual's speaking-and-thinking activities.

Turning to the information that exists in scientific sources, it is quite easy to make sure (Supervenience) that the concept of supervenience occupies the central position in modern analytical philosophy. It is also often used in the philosophy of mind to describe the dependence of mental processes on physical phenomena. If to consider its dictionary definition, the word supervenience means the action arising as a result from something else or following something.

In scientific practice, supervenience is defined as the state of any system determined by the state of another system. This also implies that the complex of properties of one system is supervenient in relation to the complex of properties of another system if the existence of a difference between the two facts in the properties of the first system is impossible without the existence of the same difference between the facts in the properties of the second system. 
If to understand the very word supervenience as a movement, as it originally means, then the indicated difference between the facts should be considered as dialectical contradictions generating this movement.

The overwhelming majority of present-day scholars in the field of cognitive studies share Donald Davidson's point of view, who stated that psychological qualities cannot be reduced to physical qualities, but they can strongly depend on them (Supervenience). Therefore, it is usually considered and said (Supervenience and Mind) that consciousness is supervenient on the brain, i.e., the physical state of the brain is primary in relation to the properties of consciousness.

Without dwelling into deeper consideration of this aspect of the issue, we shall emphasize that in our further research we will define as supervenient any deterministic relations in which a certain complex of interrelated factors having a different nature of origin is able to play the role of a cause that generates as a consequence another complex of interacting factors of different nature.

In turn, traditionally, by synergism in any cognitive process of human's speaking-and-thinking and thinking-and-acting activities, we will consider as a joint action of different in nature sources of energy aimed at achieving a specific goal by individual's psychic (cognitive) system on the basis that the energy of this system will always be greater than the sum of energies of the sources themselves.

Going deeper into the problem, it is easy to make sure that, firstly, cognition as a complex system, thanks to whose functionality the person decodes, processes, stores and uses information during his/her interaction with the surrounding reality and society, is carried out on the basis of speaking-and-thinking activities.

Secondly, in any cognitive system, it is possible to single out three interacting autonomous systems (or subsystems), namely: the system of causing factors, the energy system of cognitive processes and acts' self-development, the system of consecutive factors (the final states of the cognitive system or the results of cognition).

Thirdly, and this is especially important, the causing factors and types of energies driving the cognitive processes' self-development, and consecutive factors that take place in cognition are, as a rule, different in their nature.

Fourthly, the multifactorial nature of cognitive processes predetermines the emergence of relations between the complexes of causes and consequences that have a specific probabilistic correlative nature.

Due to the existence of these features, for our further deeper consideration of the essence of multifactorial relationship and the use of its features for methodological purposes, it is rational, in our opinion, to delve into the system of causing factors.

\section{Results and Discussions}

For this, we regard it sufficient to use the graphical image of the generalized systemic model of the factors interaction determining the individual's personality self-development (Klymenyuk 2010: 269) and by way of slightly changing the content of its elements in accordance with the aim of the analysis, to form a new, more specific cybernetic model. 
Let us point out that the following postulates were taken as a conceptual basis for the model semantic loading.

1) the human's personality formation and continuous self-development is possible only under the conditions of his/her socialization;

2) public consciousness is supervenient on public being;

3 ) the personality development, originating in the micro-society, is carried out in direct interaction with the macro-society, with an indirect relationship with the hyper-society as a supersystem that unites them;

4) synergetics of the stochastic self-development of non-equilibrium open systems lies in the basis of cognitive processes that take place in the individual's psyche;

5 ) within the interaction of noo-sphere and the sphere of individual's spiritual being, there is the law of equality of the influences exerted on the society by the individual and vice versa, by the individual upon the society.

On the basis of these postulates, we have formed a system model presenting the structure and complex interaction of causing factors that influence the cognitive processes' self-development that occur in the human psyche (see Fig. 1).

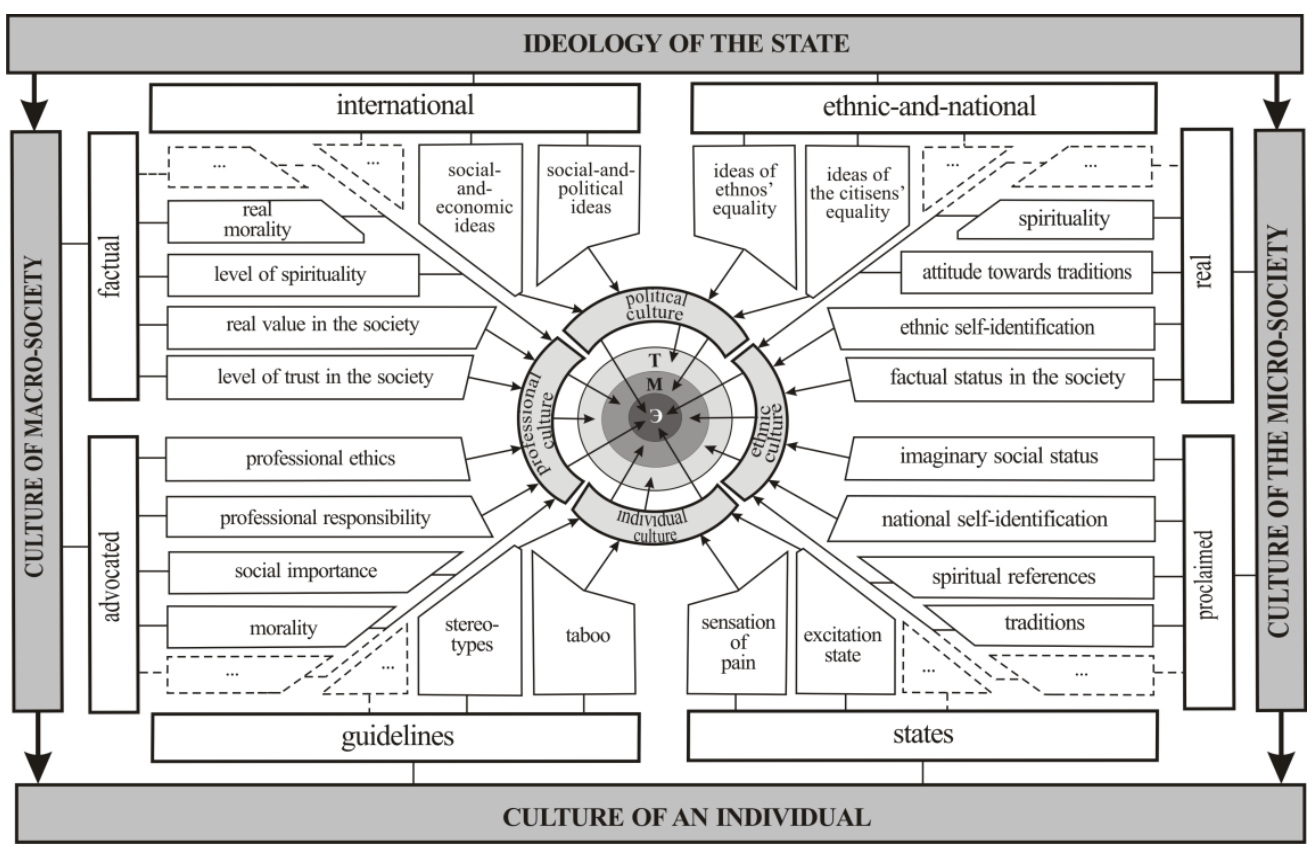

Fig. 1. System model of the structure and complex interaction of causing factors that affect the self-development of cognitive processes in the human psyche

The model embodies the idea of a synergetic mechanism of the impact of complexes of socio-cultural factors on the specificity of cognitive processes' selfdevelopment, whose results, either in an extraverted or introverted form, are actualized by the individual in his/her speaking-and-thinking activities (i. e. domaintransforming activities).

The core of the model is the sphere of the individual's spiritual being that comprises conditionally and concentrically located existential, mental and transcendental spheres of the individual's being with inherent to them emotional, emorational and rational types of thinking (Klymenyuk 2009), controlled by the 
consciousness, whose functioning results are actualized in speech in oral or activityrelated forms.

The core thus constructed reflects the cognitive unity of all processes of the individual's speaking-and-thinking activities, actualized at the levels of existence, mentality and transcendence on the basis of a common mechanism. We will not delve into the specifics of functioning and interaction of the above mentioned spheres of the individual's spiritual being since their systematic description can be found in a number of works (Klymenyuk 2010: 178-187, 276-280; Klymenyuk 2009).

We will only emphasize here that the existential being of a person, driven by emotional thinking, takes place in the sphere of his/her unconsciousness, mental being is the result of his/her emo-rational thinking, while transcendental being based on a rational type of thinking is realized in the sphere of sub-consciousness. In this case, the driving force of all types of the individual's spiritual being is his/her psycho-physiological energy.

It is perhaps worthy to note that the term existential means everything that refers to the uniqueness of intra-human being, the individual's awareness of his/her involvement in the superior, and that cannot be expressed by the language of concepts. The notion of mental presupposes the result of the deep levels interaction of collective and individual consciousness, which determine the way of person's thinking and feelings, based on his/her unconscious mental attitudes, as well as on socially acquired rational skills of emotional and behavioral readiness to perceive the surrounding world. The term transcendental denotes the acts, processes, or any product of human thinking about the super-categorical fullness of his/her existential being or some of its characteristics that are inaccessible by direct cognition and can be cognizable only speculatively, due to the fact that they can be expressed in the language of abstract notions.

It was indicated above that all cognitive processes, originating in the sphere of the individual's existential being, develop on the basis of emotional (unconscious) thinking. In this sphere, under the influence of emotional energy, the fields of intentions are formed that the person is not aware of. The energy of these fields is transferred to mental structures and, through resonant excitations of emotional and logical (rational) beginnings of the human psyche, is transformed into pragmatic intentions that the person is not fully aware of yet, which, under the control of consciousness, are transformed in his/her mental sphere into quite definite pragmatic aims or objectives.

The fundamental difference between the flow of emo-rational cognitive processes in the individual's mental sphere (i. e. in his/her subconsciousness) lies in the fact that this flow is provided by the interaction of emotional states ("emotion") and acts (beginnings) of a transcendental logical type of thinking («rational»).

As for the processes realized in the sphere of the individual's transcendental being, they are controlled by the consciousness only in relation to the general rules of their occurrence and flow. As a result of the energetic interaction with the brain material structures, being responsible for the logical beginning of thinking, the acts of transcendence are reformatted into the corresponding procedures of logical thinking, whose product materializes in verbal or activity-related forms without significant losses of psycho-physiological energy.

Unfolding similarly to the mental being in the subconsciousness, the transcendental being of a person also proceeds according to the laws of selfdeveloping chaos and is fundamentally different from its noumenal existential being 
and phenomenal mental being, since transcendental being is driven by the acts of thinking based primarily on rational logic. As a result of transcendental thinking, specific, though not sufficiently clear, rational concepts (images or symbols) are formed, which, as a rule, are transformed by the individual's consciousness into definite abstract concepts.

It is in the processes of transcendental being that structures of rational concepts fixed by the consciousness and separated from emotions are formed. Remaining only in the person's short-term memory, these concepts do not last long. But it is just due to the subconscious transcendental being which unfolds on these concepts' content basis and which is characterized by the synergetic mechanism of polylectic (Klymenyuk 2010: 147-167) rational thinking, that a person acquires his/her creative abilities.

However, the consciousness remains the main and special sphere of a person's spiritual being. The development level of the consciousness and its conceptual content determine the qualitative aspect of the individual. The consciousness as a complex psychoenergetic system develops and monitors the implementation of behavioral techniques that ensure human's survival in the almost unpredictable material and social worlds.

Figuratively speaking, to solve the strategic problems arising in the life of an individual and operational tasks they generate, the consciousness uses the results of a person's biological instinctive emotional experience (a product of existential being), genetic and cultural information (a product of mental being) and socio-cultural knowledge (a product of transcendental being).

Having briefly described the processes occurring within the four spheres of human spiritual being viewed as a typical complex synergetic system of a nonequilibrium open nature, interpreted by the core of the model in Fig. 1, we can proceed to considering the system of causing factors that affect the characteristics of these processes' synergistic self-development.

The structure of the model shows that the source of cognitive processes' selfdevelopment is the contradictions that arise both between separate causing factors and between their specific complexes. At the global level of the reality, these contradictions most often arise between the following: the official state ideology and the civilizational culture of the macro-society, the official state ideology and traditions of the microsociety, the civilizational culture of the macro-society (for instance, a professional team, Diaspora, religious community, public organization and the like) and the traditions of the micro-society (e.g., family, orphanage community, etc.). The model shows that at the global level, contradictory relations between the individual and the society can develop along six axes, meaning that their self-development has six degrees of freedom. At the same time, as the world civilization develops, the intensity and tension of these contradictions will inevitably change.

Before further reasoning, it is worthwhile emphasizing the fact that culture in its broad understanding (Prohorov 1987: 669) reflects a certain historical level of the development of a society, a person's creative forces and abilities, revealed in the types and forms of the people's life and activities organization as well as in their tangible and spiritual values.

The model also demonstrates that at the second hierarchical level, these general categorial contradictions (being of a high level of abstraction) are differentiated into more specific ones, reflecting, in fact, the struggle between the advocated and factual, declared and real, a mental set and a psychological state. 
The end points of contradictions being at the third hierarchical level of the model can be represented by the following: traditions - attitude towards them, the social significance of a profession - its real value in the society, the social position of an individual - his/her imaginary social status, religion - spirituality, national self-identification - ethnic self-identification or self-consciousness, etc.

We shall note that the limited format of the model graphic representation does not allow us to reflect upon the broader morphological set of factors that have a common origin (for instance, the advocated civilizational culture of the macrosociety or its actual culture). This necessitates the introduction of graphic symbols in the indicated sets in the form of empty dotted triangles and quadrangles, indicating the practical possibility of considering a much larger number of such factors.

Here, as in many of our works, let us pay attention to an important circumstance. It lies in the fact that due to the prevalence of utilitarian type of thinking and mercantile interests in the overwhelming majority of people, the most intense cognitive contradictions arise as a result of dialectical interaction of causing factors of a high level of abstraction (for instance, the official state ideology) and specific causing factors of their lower hierarchical level (for example, the real status of an individual in the society).

As one can see, cognitive contradictions can be formed under stochastic influence of an almost incalculable number of causing factors, different in their nature and the degree of their influence on the self-development of the individual's speaking-and-thinking processes. Taking this into account, we have developed a corresponding four-level model of the global system of complexes and individual factors that can play the role of causes that affect the specifics of cognitive processes' self-development.

Let us remind here that in synergetic systems all the varieties of complexes of causing factors that exist in reality can lead to the change in the parameters of the corresponding complexes of consequences, which, like causes, have a different nature. These supervenient complexes of consequences are called (Kurdyumov \& Knyazeva 2002: 113) the parameters of the state of the system. Naturally, it is practically impossible to carry out an adequate description of the system behavior on the basis of such an obviously excessive number of parameters of the system state. In this regard, within the framework of the synergetic approach, it is customary to use the method of information compression, whose essence is the transition from variables called the system state parameters to the parameters of order, being the functions of these variables.

The need to move to the parameters of order that integrate the influence of the most significant variables on the process of the system self-development as a whole is also preconditioned by the fact that they determine the behavior of its parts or subsystems (in our model, these are the existential, mental and transcendental spheres of the individual's spiritual being).

Let us pay attention to the fact that the complex and individual factors indicated, respectively, at the second and third hierarchical levels of the model (Fig. 1), refer to the parameters of the state of the cognitive system under study. At the same time, in the most general consideration, the role of the parameters of order can be fulfilled by the following complex of causing factors, namely: state ideology, culture of the macrosociety, culture of the micro-society, and the individual's culture. 
In other words, in a global consideration as the parameters of order, the characteristics of speaking-and-thinking processes' self-development that take place in the spiritual sphere of the individual's being will always be influenced by cultures as a whole: those of the state, the macro-society, the micro-society and the individual's ones.

It should be kept in mind that, firstly, according to the laws of dialectical methodology, the driving force of any stage of cognitive self-development can only be the contradictions arising between the two energetically most active conceptopposites (the parameters of order in our case) generated by the development levels of these cultures. Secondly, the third parameter of order in the cognitive systems of an individual's speaking-and-thinking activities is always his/her pragmatic intentions, which excite the energy of emotional states necessary for their selfdevelopment. Thirdly, in cases of multi-stage cognition, the two parameters of order of its self-development at each subsequent stage usually change in the direction from abstract to concrete or vice versa, and thus pragmatic attitudes are slightly corrected. Fourthly, the choice of two dialectically opposing parameters of order is determined, basically, by the aim of the research and is limited by its object.

It should also be emphasized that in accordance with our model, at the global level (i.e. the level of maximum abstraction of causing factors), it will be completely sufficient for solving any tasks of a cognitive researcher, to regard any of the following six contradictory pairs as the parameters of order, namely: state ideology the culture of the macro-society; state ideology - the culture of the micro-society; state ideology - personality's culture; the culture of the macro-society - the culture of the micro-society; the macro-society culture - personality's culture; the microsocial culture - personality's culture.

In other situations, the researcher, in accordance with the study objectives, is free to choose the parameters of order out of their potentially infinite set, reflected in the model on Fig. 1.

Thus, for instance, in the case of considering the problems arising within the civilizational culture of the macro-society, it is quite enough to choose the advocated culture and the actual culture of the macro-society as the parameters of order. Then, from the point of view of cybernetics, such causing factors (indicated in the model on Fig. 1) as professional ethics, professional responsibility, social significance, morality, etc., as well as the level of trust in the society, real values in the society, the level of spirituality, real morality, etc. will play the role of disturbing influences entering the cognitive system under study. In this case, the function of the control action will remain with the three parameters of order, while maintaining the dominance of the pragmatic attitude as the goal of the system self-development. Such consideration will result in the description of the real state of the macro-society culture (in our example, the professional culture), and for the individual as a subject of cognition - the renewal of the individual picture of the social world.

The study of cognitive processes becomes more complex when the leading causing factors and the results of their influence are fundamentally different in the nature of their origin. On our model, the parameters of order of such systems' selfdevelopment can be the following: the state ideology and personality's culture, the macro-society culture and personality's culture, the micro-society culture and personality's culture. On the one hand, the complexity of such cognitive systems forces the researcher not to lose out of sight the worldview (or behavioral) attitudes 
and taboos, as well as the emotional and physical states of a person, in whose psyche there is a self-development of speaking-and-thinking processes. On the other hand, within the field of such parameters of order, the researcher regards the cognition problems associated with the personality's self-development (for instance, its formation in children). At the same time, the issues of synergetic interaction in cognition of different in their origin types of energy require a special approach to the study within the framework of lingua-energetic aspect.

One cannot but take into account the fact that our model reflects a feature of the objective reality, formulated in the third postulate, according to which the selfdevelopment of the individual's personality inevitably occurs under the continuous dominating influence of the cultures of hierarchically subordinate societies, since the ideology severely affects the cultures of macro- and micro-societies, and they, in their turn, produce strong influence on the individual's culture.

In this regard, let us consider an example of modeling cognition, whose selfdeveloping nature is influenced by such global parameters of order adopted by the researcher as the advocated culture of the micro-society and the individual's inner culture. For this let us imagine that the boss, in the presence of colleagues, made an unjustly rude remark to his employee in an unacceptable form, thereby violating the requirements of professional ethics. The psychological reaction of the subordinate aroused definite personal attitudes in his consciousness that were formed as a result of his previous social experience. It is clear that the direction of further cognitive processes' self-development in the system of his spiritual being will, primarity, be determined by such parameters of order as the norms of professional ethics, advocated by the society, and the individual's personal attitudes, which generate certain stereotypes of behavior in his mind.

It is also easy to imagine the feelings an offended employee should experience in a state of emotional excitement. Most likely, these will be the feelings of humiliation, resentment, fear, indignation, protest, anger, hatred, etc., which inevitably arise in the sphere of any individual's spiritual being.

However, one should undoubtedly expect a very restrained verbal reaction of the subordinate, who rarely manages to hide his feelings expressed by the non-verbal behavior (facial expressions, gestures, posture, etc.). This can often occur due to a personal attitude (e.g. the stereotype he has assimilated that «the boss is always right») or an inner taboo on conflict behavior, rooted to the level of the instinct for survival in the society and even deeper than that - the level of his self-preservative instinct.

Being very typical, the described situation should not, nonetheless, be considered as an obligatory regularity, since this issue in each specific case should be approached with special attention, taking into account the pendulum nature of the human mental sphere reaction to the irritating factors, which are called in synergetic systems as disturbing influences. Therefore, in such situations, the individual's natural reaction to the pressure on him by the cultural bans can, generally speaking, take two forms: the form of and open protest and its sublimated variants, which are various forms of latent (inner) protests.

In reality, more often the forms of inner protest that are more difficult to study are actualized, first of all, due to the peculiar appearance of a powerful survival instinct, supported by the imitative essence of the psychic processes of the individual's personality formation. 
But irrespective of the cognitive processes proceedings, the methodological requirements for the completeness of their interdisciplinary lingua-energetic qualitative and quantitative description remain unchanged. To do this, it is necessary to be able to describe at least two regularities: the changes in the qualitative characteristics of the cognitive system, integrated into the concepts points' content basis, and the fluctuations of psycho-physiological energy that generates certain emotional experiences and corresponding feelings. We shall note that at present cognitive science has (Kalyta 2016: 169-181, 196-203) methods and technical means of such a description of the results of the individual's speaking-and-thinking activities materialized in his/her behavior and speech.

\section{Conclusions}

The situation that has developed in such a way unambiguously allows us to use the formed systemic model as a methodological tool for planning and conducting the interdisciplinary lingua-cognitive research aimed at studying the myriad of new energetic phenomena of human speaking-and-thinking activities.

On the basis of the mentioned above, it is not difficult to understand that the model allows one to foresee the possibility of planning a systematic study of statistical and correlation patterns of the psycho-physiological energy consumption of an individual's cognitive processes self-development caused by contradictions between: specific ideological attitudes of the state and the individual's personal culture; morality promoted in the macro-society and the real spirituality of the micro-society surrounding the individual; the state ideology and the individual's macro-society culture; the individual's social significance promoted by the society and his/her actual status in the society; morality and real attitude of the individual to the ethnic traditions; the requirements of professional ethics and morality of the individual, etc.

And this is just a small part of the scientific problems, potentially encoded in the methodological essence of the systemic model of a complex interaction of causing factors that affect the cognitive processes' self-development in the human psyche. In order to feel and practically use the potential infinity of the variety of topics and problems of scientific cognition latently embedded in the model, an attentive researcher needs to mentally move to the lower hierarchical level of synergy of contradictions, i. e. to the level of real interaction of instincts, feelings and their superveniently diverse complexes.

Performing a broader global analysis of cognitive problems up to the issues of transformation of supervenient correlations or other forms of connections between the elements of the four autonomous systems indicated in the model, it is advisable to consider them as one mega-system including, respectively, the hyper-system of the state ideology, the systems of cultures of macro- and micro-societies and the subsystem of the individual's culture.

As for the lingua-energetic analysis of speech, within its framework it is reasonable to consider the behavior and interaction of the three conditionally autonomous energetic systems: physiological, cognitive (psycho-physiological) and physical (physiological-acoustic). 
In conclusion, we shall remark that since the world of ideas about cognitive phenomena is extremely hypothetical, and the hypotheses themselves are very controversial, then, at the interdisciplinary level of research, it is necessary, in our opinion, to pay special attention to the timely figurative and terminological unification of the acquired knowledge based on intermediate, albeit not always academically impeccable hypotheses and ideas, because we do not have any other more effective methodological tool at our disposal for increasing scientific knowledge.

\section{References}

Kalyta, Alla. 2016. Speech energetics. Kyiv: Kafedra.

Kalyta, Alla., Klymenyuk, Oleksandr. (2019). "Theoretical and methodological grounds of studying the fluctuation of the emotional-and-pragmatic potential of the individual's speaking-and-thinking and thinking-and-acting activities". Odessa Linguistic Journal, 13: 115-123.

Klymenyuk, Oleksandr. 2005. Methodology and methods of a scientific research. Kyiv: Millennium.

Klymenyuk, Oleksandr. 2009. "Models of conceptual spheres and mechanisms of a person's cognitive thinking”. Scientific notes, 81(3): 88-104.

Klymenyuk, Oleksandr. 2010. Knowledge, learning, cognition. Ternopil: Pidruchnyky i posibnyky.

Kurdyumov, Sergei., Knyazeva Yelena. 2002. "The structures of the future: synergetics as a methodological basis of futurology". Synergetic paradigm. Nonliner thinking in science and art. Moscow: Progress Tradition.

Prohorov, Aleksandr. 1987. Soviet encyclopedic dictionary. Moscow: Soviet encyclopedia.

Supervenience. Retrieved from: https://www.britannica.com/topic/supervenience

Supervenience and Mind. Retrieved from: https://iep.utm.edu/supermin/ 